\title{
Effects of Respiratory Rate on Heart Rate Variability in Neurologic Outpatients with Epilepsies or Migraine: A Preliminary Study
}

\author{
Katrin Druschky ${ }^{a, b}$ Jürgen Lorenz ${ }^{c}$ Achim Druschky ${ }^{a, b}$ \\ ${ }^{a}$ Neurologische Gemeinschaftspraxis, PDDr. Katrin Druschky, PDDr. Achim Druschky, Nürnberg, Germany; \\ ${ }^{b}$ Department of Neurology, University of Erlangen-Nuernberg, Erlangen, Germany; ${ }^{~}$ Faculty of Life Science, \\ Laboratory of Human Biology and Physiology, Applied Science University, Hamburg, Germany
}

\section{Highlights of the Study}

- Broad variations in spontaneous respiratory rates were found in 2 groups of patients with neurological diseases common in outpatients.

- Respiratory rate was negatively correlated with heart rate variability (HRV).

- Differences in HRV were significantly lower during slow compared to fast breathing.

- Simultaneous measurement of breathing rates is important for the interpretation of HRV parameters in patients.

\section{Keywords}

Spontaneous respiratory rates · Heart rate variability ·

Migraine $\cdot$ Epilepsy Paced breathing

\begin{abstract}
Background: Variation of spontaneous respiratory rates and influences of spontaneous and paced breathing rates on heart rate variability (HRV) were assessed in patients with epilepsy or migraine, and HRV parameters were compared between the groups. Materials and Methods: Thirty neurologic outpatients, 16 diagnosed with epilepsies and 14 with migraine, were included. Autonomic testing consisted of short-term HRV, the deep breathing test (DBT), and measurement of HRV at systematically changed breathing rates (paced breathing, 5-18 breaths per minute, bpm). Results: Spontaneous respiratory rate during short-term HRV varied
\end{abstract}

from 9 to $23 \mathrm{bpm}$ in the epileptic group and from 5 to $21 \mathrm{bpm}$ in migraine patients and was significantly and negatively correlated with SD of all normal RR intervals (SDNN) and total power (TP) in epileptic patients but not in migraine patients. Paced breathing rate had a significant effect on all HRV parameters assessed in both groups. HRV (SD1, SDNN, TP) and DBT (E-I, SD1, SDNN) parameters were significantly lower in the epileptic group. Group differences were significantly greater during slow compared to fast breathing. Conclusions: An important and new finding is the wide variation of spontaneous respiratory rate in both groups, along with the significant negative correlation with the assessed HRV parameters. The reduction of HRV during slow breathing in epileptic patients may indicate a diminished cardiorespiratory coupling caused by a probable loss of sensitivity within the cardiovagal brainstem circuitry.

(c) 2019 The Author(s)

Published by S. Karger AG, Basel

\begin{tabular}{ll}
\hline KARGER & $\begin{array}{l}\text { Ko } 2019 \text { The Author(s) } \\
\text { Published by S. Karger AG, Basel }\end{array}$ \\
karger@karger.com & This is an Open Access article licensed under the Creative Commons \\
www.karger.com/mpp & $\begin{array}{l}\text { Attribution-NonCommercial-4.0 International License (CC BY-NC) } \\
\text { (http://www.karger.com/Services/OpenAccessLicense), applicable to } \\
\text { the online version of the article only. Usage and distribution for com- } \\
\text { mercial purposes requires written permission. }\end{array}$
\end{tabular}

Katrin Druschky

Neurologische Gemeinschaftspraxis

PDDr. Katrin Druschky, PDDr. Achim Druschky

Am Stadtpark 2, DE-90409 Nürnberg (Germany)

E-Mail info@druschky.de; katrin@druschky.de 


\section{Introduction}

Epilepsies and migraine are frequent diseases of neurologic outpatients. Autonomic dysfunction has been described in patients with these diseases [1,2]. Several autonomic function tests can be used to noninvasively evaluate the severity and distribution of autonomic failure [3], which can be grouped into 3 general categories of autonomic activity: cardiovagal tests, adrenergic tests, and sudomotor tests [4].

In the time domain, the commonly used and most reliable approach for cardiovagal function testing is to quantify the heart rate (HR) response to deep breathing and to the Valsalva maneuver [3]. During the deep breathing test (DBT), the subject breathes at $6 \mathrm{bpm}$, and the magnitude of $\mathrm{HR}$ variation that occurs during a respiratory cycle (maximal HR with inspiration minus minimum HR during expiration) is averaged (E-I) and provides an index of cardiovagal function [3].

Cardiovagal function can also be evaluated in the frequency domain using spectral analysis of resting HR. Resting HR variability (HRV) is derived from changes in RR intervals in a continuous ECG recording. Total power $\left(\mathrm{TP} ; \mathrm{ms}^{2}\right)$ corresponds to the area under the entire power-spectral curve and reflects the total variance of all normal RR intervals.

Within the TP range, low-frequency (LF) and highfrequency (HF) bandwidths are estimated, ranging from 0.04 to $0.15 \mathrm{~Hz}$ and from 0.15 to $0.4 \mathrm{~Hz}$, respectively. $\mathrm{HF}$ power is strongly coupled to respiration, giving rise to the well-defined respiratory sinus arrhythmia (RSA). RSA is believed to reflect primarily vagal activity because the bronchial and thoracic pressure sensors convey their afferent signal to the nucleus tractus solitarii via the vagal nerve. The synaptic relay from nucleus tractus solitarii to the nucleus ambiguous represents the origin of the efferent pathway of the vagal nerve to the sinoatrial node. Therefore, heart beat slows during expiration when positive intrapulmonary pressure excites the pressor receptors and heart beat increases during inspiration when pressor sensor input decreases. In contrast, LF parameters are assumed to reflect a combination of vagal and sympathetic activity. A major contribution of the LF band power reflects the periodicity of around $0.1 \mathrm{~Hz}$ of the baroreceptor reflex arc, originating from pressor receptors located in the aorta and carotis bifurcation regulating arterial blood pressure. During deep slow breathing at 6 cycles per minute, which corresponds to a frequency of $0.1 \mathrm{~Hz}$, the RSA is maximally elicited, partly because it synchronizes with the baroreflex frequency [5]. Increased

Effects of Respiratory Rate on HRV breathing rate is accompanied by a reduction of RSA [6]. At more natural breathing rates between 10 and 24 cycles per minute, HF power is clearly distinct from LF power allowing to estimate the LF-to-HF ratio, often referred to as indicator of the sympathovagal balance.

In the time domain, the square root of the mean squared differences of successive RR intervals and the proportion derived by dividing the number of interval differences of successive RR intervals $>50 \mathrm{~ms}$ by the total number of RR intervals (pNN50) are considered measures of cardiovagal function, whereas the SD of all normal RR intervals (SDNN) reflects the global index and thus overall autonomic cardiac activity. Square root of the mean squared differences and SD1, the latter being a Poincare plot component, are identical metrics of HRV [7]. In Poincare plots, the heart period $\left(\mathrm{X}\right.$-axis $\left.=\mathrm{RR}_{\mathrm{i}} \mathrm{ms}\right)$ is plotted against the subsequent heart period ( $\mathrm{Y}$-axis = $\left.\mathrm{RR}_{\mathrm{i}+1} \mathrm{~ms}\right)$. SD1 is an index of "short-term" HRV and can be understood as the SD of the perpendicular distances between each $\mathrm{X}-\mathrm{Y}$ coordinate and the line of identity in the Poincare plot.

Compared to standard laboratory function tests, which evaluate HRV under modified physiological parameters, investigations centered on resting HRV are relatively easy to perform. Nevertheless, up to now, the clinical relevance of HRV has been clearly demonstrated in only 2 clinical conditions. In the late 1980s, it was confirmed that HRV was a strong independent predictor of mortality after acute myocardial infarction [8] and an objective method for the detection of peripheral diabetic neuropathy [9].

Measurements and analyses of resting HRV are commonly done without consideration for respiratory parameters such as depth and frequency, despite their wellknown influence on both HR and HRV, independent of cardiac autonomic activity. Systematic investigations on the influence of breathing patterns on resting HRV are rather scarce and almost exclusively limited to studies in healthy volunteers. With very few exceptions, for example, in patients with severe mental disorders [10], there are no such data available from patient investigations.

The present study was designed to establish (1) the range of spontaneous respiratory rates during resting HRV measurements in 2 diagnostic groups of neurologic outpatients (epileptic and migraine patients), (2) whether and to what extent predefined ascending and descending breathing rates during paced breathing influence HRV, and (3) whether the 2 diagnostic groups of epileptic and migraine patients differ in HRV at rest and during deep breathing. 


\section{Methods}

\section{Study Population}

Patients were recruited in a neurological group practice in Nuremberg, Germany. The following inclusion criteria were applied: neurological diagnosis of migraine or epilepsy (diagnostic criteria given below), voluntary participation, minimum age 18 years old, and capacity for consent. Exclusion criteria included diagnoses of dementia or cognitive impairment as well as inability to participate in the autonomic testing.

Thirty participants, 16 diagnosed with epilepsies and 14 with migraine, were included in the study. The group of epileptic patients consisted of 7 females and 9 males, aged 19-66 years (mean $=39$, SD 14.6). The group of patients with migraine included 11 females and 3 males, aged 19-47 years (mean 30, SD 7.6). Epilepsies and migraine were diagnosed by history, neurological examination, EEG, and cranial magnetic resonance imaging (to rule out symptomatic causes). History included the mean number of seizures or migraine attacks per month or year, medications taken, previous diseases, regular physical activity (hours per week), and consumption of tobacco and alcohol. Patients with migraine were diagnosed according to the diagnostic criteria for migraine of the International Headache Society classification . Epilepsies were diagnosed according to the guidelines of the Deutsche Gesellschaft für Neurologie and ILAE classification of the epilepsies. Physical examination included measurement of the weight and height for the calculation of the body mass index (BMI).

All patients gave written informed consent. Study-related data were stored anonymously. The study was approved by the local Ethics Committee.

\section{Experimental Protocol}

Study examinations included psychological screening using Beck's Depression Inventory (BDI) and the Perceived Stress Scale (PSS-10).

Autonomic testing included the measurement of 5-min shortterm HRV, 2-min HRV during deep breathing (at $6 \mathrm{bpm}$ ), and 9-min HRV at systematically changed breathing frequencies (paced breathing). To account for possible habituation of RSA over time, during paced breathing, 5 breathing rhythms were employed (every $60 \mathrm{~s}$ ), in ascending and descending sequence $(5,6,8$, $12,18,12,8,6,5 \mathrm{bpm})$, resulting in a total recording time of $9 \mathrm{~min}$.

\section{Methods of Analysis}

Autonomic function data were analyzed using the HRV-Scanner software developed by Biosign ${ }^{\circledR}$ ("HRV-Scanner"; Biosign ${ }^{\circledR}$, D-85570, Ottenhofen, Germany). The device offers a standard 3-lead electrocardiogram (ECG) with a sampling rate of $1 \mathrm{kHz}$ and 16-bit resolution. Filtering of artifacts and ectopic heart beats was done automatically by a patented algorithm in the phase space by means of the analysis of a Poincare plot, where ectopic heart beats and artifacts can easily be detected as outliers. In a second step, an experienced investigator assessed the resulting HR curve for remaining artifacts. HRV analysis was conducted according to actual guidelines [11]. Spectral analysis was performed by means of FFT analysis on the $4 \mathrm{~Hz}$ cubic spline interpolated curve of RR intervals.
Measurements of Respiratory Rates

Respiratory rates were determined via chest belt as well as calculated from the ECG registration as previously described [12,13]. Corresponding hardware and software were included in the HRVScanner $\left(\right.$ Biosign $\left.^{\circledR}\right)$.

Chest belt registration of respiratory rates was done by a piezoelectric sensor at a sampling rate of $50 \mathrm{~Hz}$, with 24-bit resolution, automatic detection of breathing cycles by means of a validated algorithm, visual control of the breathing signal, and detected breathing cycles by an experienced investigator.

ECG-derived respiration was performed according to the following steps: (1) determination of each R-wave's size; (2) cubic spline interpolation of the resulting curve at $5 \mathrm{~Hz}$ using a bandpass filter (low cutoff: $0.05 \mathrm{~Hz}$; high cutoff $0.6 \mathrm{~Hz}$ ); (3) automatic detection of breathing cycles by means of a validated algorithm; and (4) visual control of the breathing signal and detected breathing cycles by an experienced investigator.

\section{Statistical Analysis}

The distribution of spontaneous respiratory rates was analyzed using descriptive statistics. From the short-term HRV measurement, we analyzed HR mean, SD1, SDNN, and TP by means of correlation analysis and multiple regression analysis to investigate the influence of spontaneous respiratory rates on HRV in both groups of patients.

The influence of paced breathing rates on HRV was investigated by repeated-measures analysis of variance (ANOVA). We tested the between-subject effect of diagnostic group (epilepsies vs. migraine) with 2 within-subject effects (period [ascending vs. descending breathing rates] and respiratory rate [5, 6, 8, 12, $18 \mathrm{bpm}]$ ) using a $2 \times 2 \times 5$-factorial design of ANOVA. Degrees of freedom for main and interaction effects with factor levels $>2$ were adjusted by the Greenhouse-Geisser epsilon. Sources of significant F values were explored by paired and unpaired $t$ tests.

Differences in HRV between epileptic and migraine patients during short-term HRV and deep breathing (at $6 \mathrm{bpm}$ ) were investigated by comparisons of $\mathrm{HR}$ mean, SD1, SDNN, and TP (short-term HRV) and by comparisons of E-I, HR mean, SD1, and SDNN (deep breathing) using the Mann-Whitney U test.

Statistical analysis was done with the statistical software STATISTICA for Windows version 10.0 (StatSoft, Inc.). Statistical significance was assumed with an error probability $p<0.05$.

\section{Results}

\section{Patient Characteristics}

Table 1 summarizes the main patient characteristics (age, gender, BMI, classification of seizures/migraine, frequency of seizures/migraine attacks) along with the results of the psychologic screening tests (BDI, PSS) and medications taken.

\section{Variation of Spontaneous Respiratory Rates during Resting HRV}

Spontaneous breathing rates during resting HRV varied considerably in both groups of patients (Fig. 1). In the epileptic group, the mean breathing rate was $15.1 \mathrm{bpm}$ (me- 
Table 1. Patient characteristics, psychologic screening tests, and medications

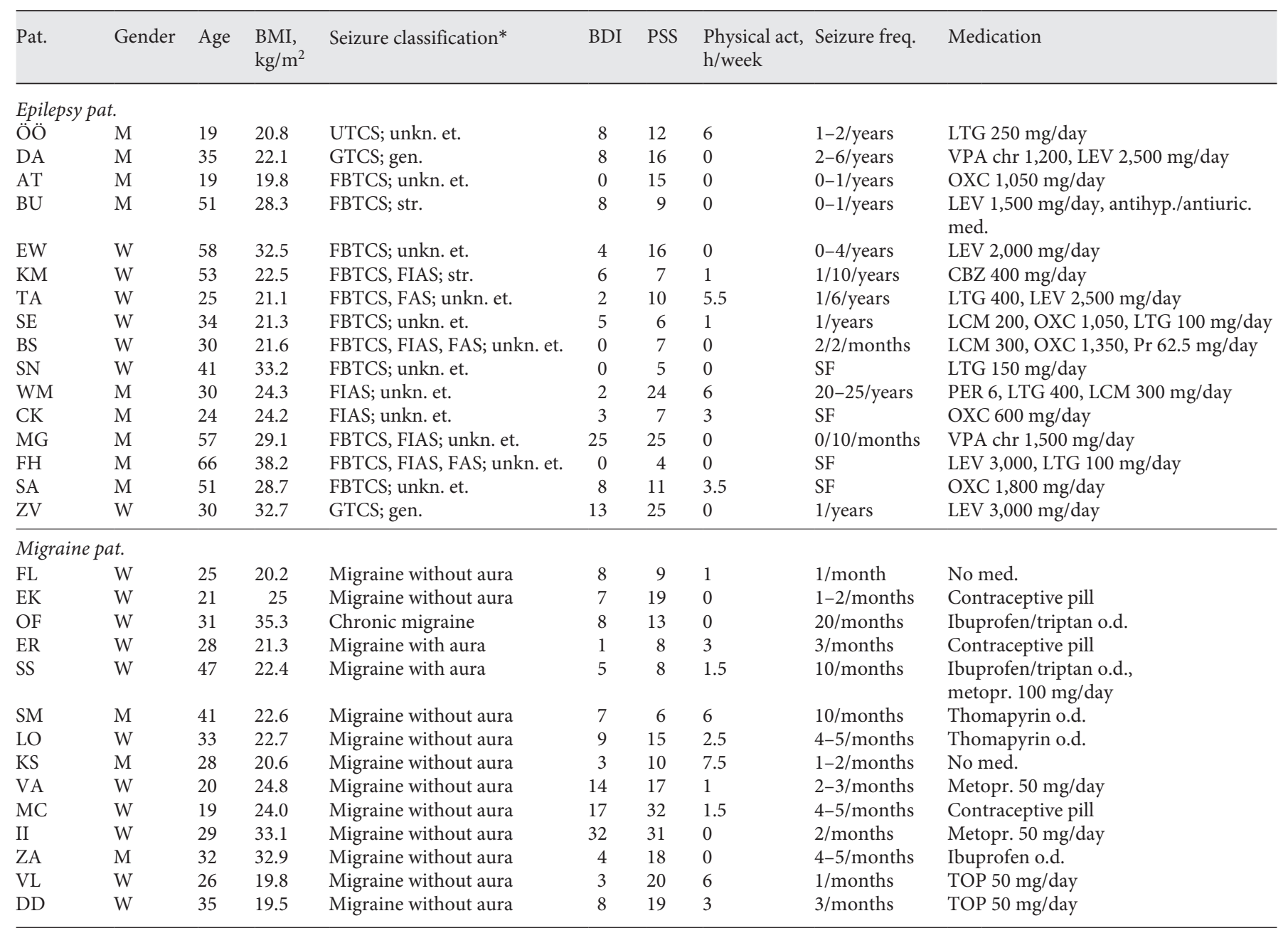

* ILAE classification of the epilepsies (Fisher et al. 2017); ** IHS classification ICHD-3.

Pat., patient(s); BDI, Beck Depression Inventory; PSS, Perceived Stress Scale; act, activity; BMI, body mass index; freq., frequency; UTCS, unknown onset tonic-clonic seizure; GTCS, generalized tonic-clonic seizure; FBTCS, focal-to-bilateral tonic-clonic seizure; FIAS, focal impaired awareness seizure; FAS, focal aware seizure; et., etiology; str., structural; LTG, lamotrigine; VPA, valproic acid; chr., chrono; OXC, oxcarbamazepine; LEV, levetiracetam; CBZ, carbamazepine; Pr, primidone; TOP, topiramate; LCM, lacosamide; PER, perampanel; SF, seizure-free under antiepileptic drug(s); antihyp., antihypertensive; antiuric., antiuricosuric; med., medication; o.d., on demand; metopr.:, metoprolol.

dian $16.1 ; \min 9.0 ; \max 23.1 ;$ SD $4.1 \mathrm{bpm})$. In the group of patients with migraine, the mean breathing frequency was $15.5 \mathrm{bpm}$ (median 15.3; min 5.3; $\max 21.4$; SD $4.6 \mathrm{bpm}$ ).

\section{Influence of Spontaneous Respiratory Rates on HRV}

Correlation analysis revealed negative correlations between respiratory rates and both SDNN and TP in both groups of patients, though these did not reach statistical significance.

To uncover potential masking effects, a stepwise forward multiple regression analysis with SDNN and TP as dependent variables was performed. Age, BDI, PSS, phys- ical activity, BMI, and respiratory rate were the independent variables. Significant $(p<0.05)$ parameters were added to the regression model. Respiratory rate had a significant negative effect on SDNN $(p=0.03)$ and TP $(p=$ 0.01 ) in epileptic patients but not in migraine patients.

\section{Influence of Paced Breathing Rates on HRV}

The influence of paced breathing rates on HRV parameters (HR mean, SD1, SDNN, TP) was investigated with ascending and descending breathing frequencies $(5,6,8$, $12,18,12,8,6,5 \mathrm{BrPM}$ ), as shown by Figure 2 . Analysis of HR and HRV during paced breathing in the epilepsy and 

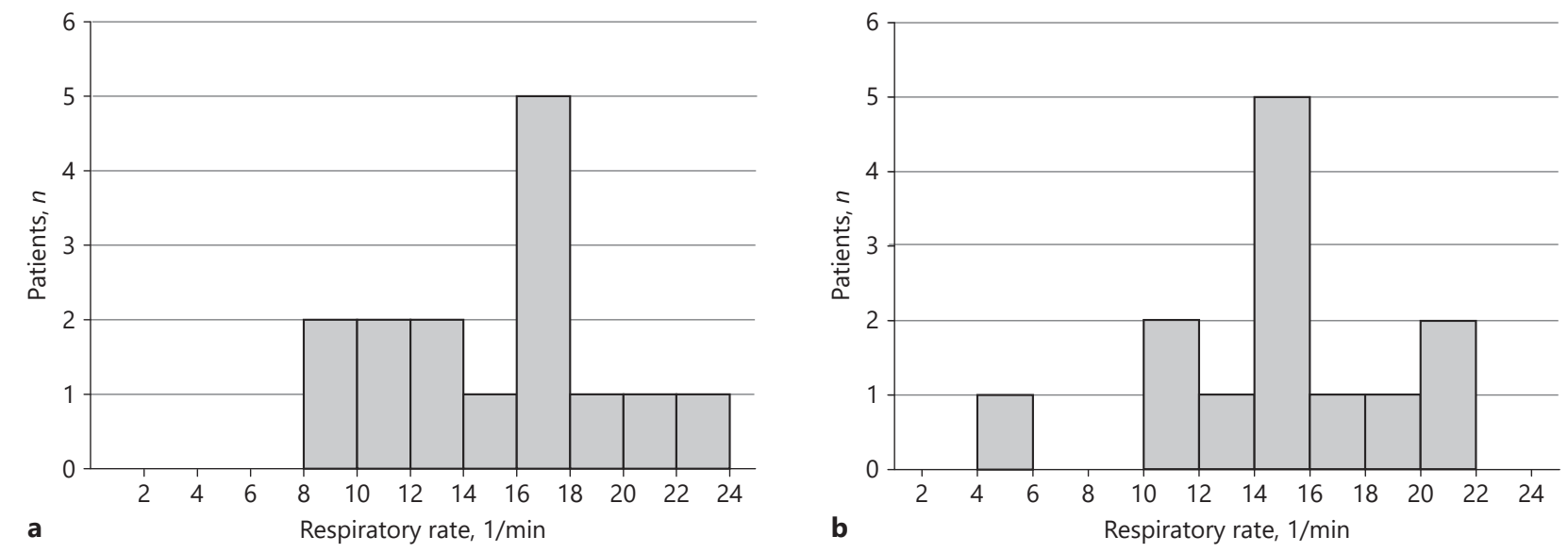

Fig. 1. Distribution of respiratory rates during short-term HRV. a Epileptic patients. b Migraine patients.

migraine groups revealed highly consistent results with respect to the influence of respiratory rate on HRV. Figure 2 illustrates the effects of respiratory rates and breathing period (ascending, descending) on the above HRV parameters in the 2 diagnostic groups. Table 2 summarizes the results of the ANOVA. Increasing respiratory rates were consistently associated with increasing HR and decreasing values of the assessed HRV parameters.

\section{Habituation Effects}

Some degree of habituation was observed with regard to SDNN, as it showed smaller values during descending $(58.2 \mathrm{~ms})$ compared to ascending $(62.1 \mathrm{~ms})$ breathing periods $(t=-2.42 ; p=0.02)$, whereas no habituation effects were seen in the other HRV parameters during paced breathing.

\section{Group Differences between Patients with Epilepsies and Migraine}

Differences in physical characteristics (age, BMI, sport activity), psychological screening tests (BDI, PSS), and short-term HRV and DBT parameters are shown in Table 3. No significant differences in the assessed physical or psychological data were observed between epileptic and migraine patients. While HR mean and spontaneous respiratory rates during short-term HRV did not reveal significant differences, the other HRV and DBT parameters studied were significantly lower in the group of epileptic patients compared to the group of migraine patients.

HRV parameters were significantly smaller in the patients with epilepsies, while differences in HR mean were not statistically significant. To illustrate the significant group-by-respiratory rate interaction during paced breathing, we averaged values over ascending and descending breathing periods, revealing lower values of HRV parameters in epileptic patients during slow than fast breathing.

To correct for possible bias caused by statistically nonsignificant age differences between both groups (higher mean age in the group of epilepsies), a stepwise forward multiple regression analysis was performed that revealed only the respective HRV and DBT parameters as significant factors.

\section{Discussion}

The present study explored the use of HRV analysis for the evaluation of autonomic dysfunction in 2 common diagnostic groups of neurologic outpatients, epilepsies, and migraine. Emphasis was placed on the influence of the respiratory rate on the examined parameters. Spontaneous breathing frequencies considerably varied within a range of 9-23 bpm in the group of epileptic patients and from 5 to $21 \mathrm{bpm}$ in the migraine group. The negative effect of higher respiratory rates on decrease of HRV parameters with higher respiratory rates was confirmed both during spontaneous and paced breathing. Slowing the spontaneous respiratory rate toward the specific natural frequency of the cardiovascular system around $6 \mathrm{bpm}$ provides a physiological increase of HRV by coupling RSA with the baroreflex cycle [5]. With increasing 


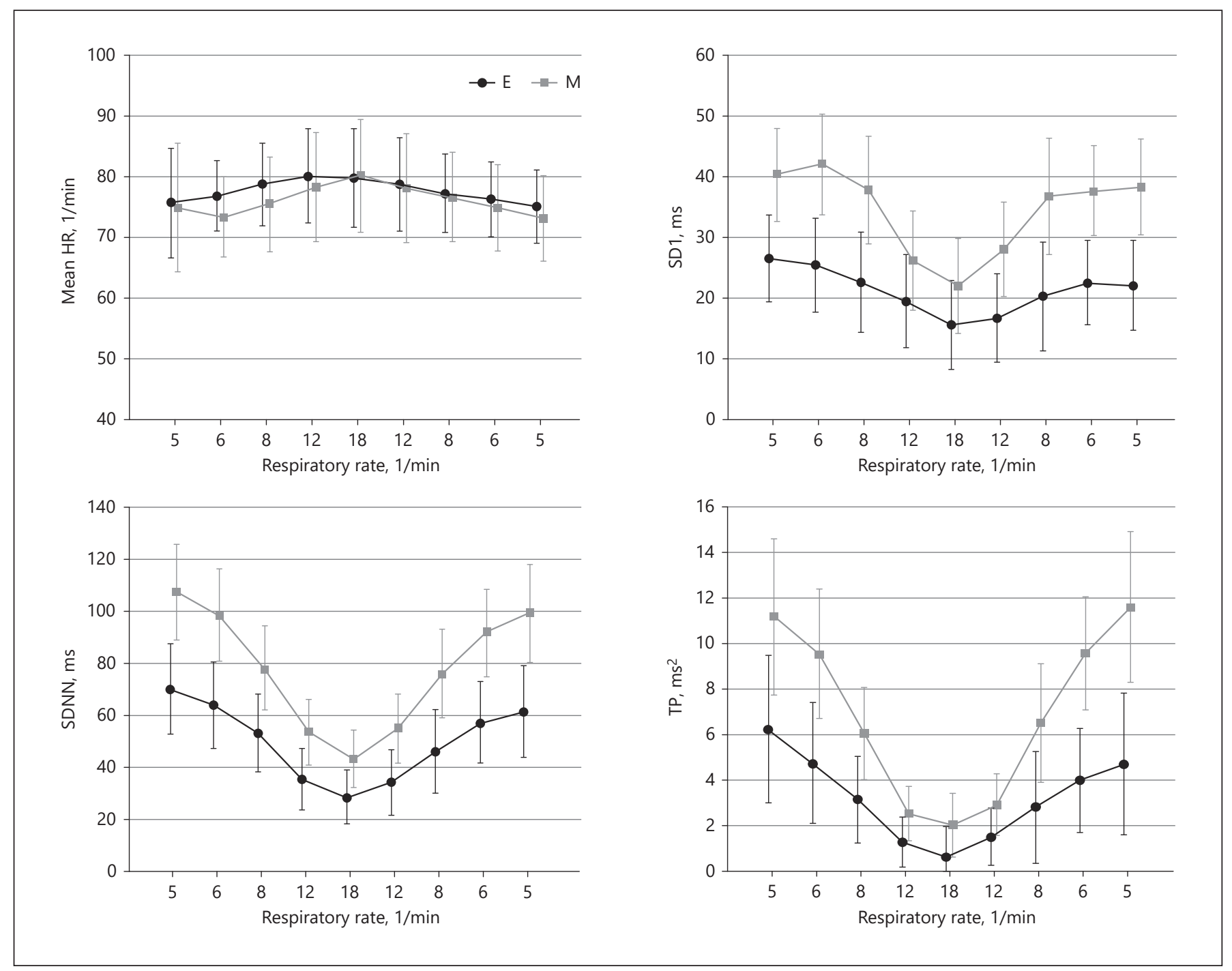

Fig. 2. Influence of paced breathing rates on HRV parameters. The error bars represent the SD. E, patients with epilepsies; M, patients with migraine; TP, total power; SDNN, SD of all normal RR intervals; HR, heart rate.

breathing rates up to the subject's natural frequency (i.e., 12-20 bpm), HRV parameters decrease, and this decrease will further continue with rising breathing rates above the normal rate, as is the case with certain diseases or in stress situations. Thus, our results support the notion that HRV results may involve disease- or stress-related confounding factors if we do not control for the respiratory rate.

The stronger reduction of predominantly vagally mediated HRV during slow and deep breathing in our group of epileptic patients points toward possible dysfunction within the cardiovagal circuitry. Disturbances of cardiac and respiratory physiology, as well as dysfunction of the autonomic nervous system, are all well-documented se- quelae of chronic epilepsy. According to a recent review of $H R$ variability in epilepsies, HF values are significantly reduced in epileptic patients compared to controls, while no such differences are observed for LF values [14]. The reduced parasympathetic activity of patients with drugresistant epilepsies has been suggested as a risk factor for sudden unexpected death in epilepsy [15].

The results from HRV studies in patients with migraine are inconsistent. According to a recent review by Miglis [16], sympathetic impairment is more common than parasympathetic impairment and more pronounced in migraine with aura. Comparing HRV parameters in patients with migraine and healthy control subjects, LF in 
Table 2. Results of the ANOVA for repeated measurements to assess the influence of diagnostic group (epilepsy, migraine) respiratory rate $(5,6,8,12,18 \mathrm{bpm}$ ) and period (ascending, descending) on HR and HRV parameters SD1, SDNN, and TP

\begin{tabular}{|c|c|c|c|c|c|c|c|}
\hline HRV Par. & Effect & F value & $p$ value & HRV Par. & Effect & F value & $p$ value \\
\hline \multirow[t]{4}{*}{ HR } & GROUP (GR) & 1.89 & 0.18 & \multirow[t]{4}{*}{ SDNN } & GROUP (GR) & 8.49 & 0.007 \\
\hline & $\mathrm{RR}$ & 30.74 & $<0.001$ & & $\mathrm{RR}$ & 76.92 & $<0.001^{\mathrm{a}}$ \\
\hline & PERIOD & 0.004 & 0.95 & & PERIOD & 5.53 & 0.026 \\
\hline & PERIOD $\times$ GR & 0.08 & 0.78 & & PERIOD $\times$ GR & 0.24 & 0.628 \\
\hline \multirow{3}{*}{ SD1 } & $\mathrm{RR} \times \mathrm{GR}$ & 3.99 & $0.034^{\mathrm{a}}$ & \multirow{3}{*}{$\mathrm{TP}$} & $\mathrm{RR} \times \mathrm{GR}$ & 5.57 & $0.015^{\mathrm{a}}$ \\
\hline & PERIOD & 2.73 & 0.11 & & PERIOD & 0.097 & 0.758 \\
\hline & PERIOD $\times$ GR & 0.40 & 0.53 & & PERIOD $\times$ GR & 1.13 & 0.296 \\
\hline
\end{tabular}

${ }^{a}$ Degrees of freedom (df) corrected by Greenhouse-Geisser. Significant effects written in bold.

Par., parameter; HR, heart rate; GROUP, diagnostic group; RR, respiratory rate; TP, total power.

Table 3. Differences in physical data, psychological screening tests, and short-term HRV and DBT parameters between epileptic (E) and migraine (M) patients as analyzed for significance by the Mann-Whitney U test

\begin{tabular}{lllll}
\hline Assessment & Parameter & $\begin{array}{l}\text { Rank } \\
\text { sum E }\end{array}$ & $\begin{array}{l}\text { Rank } \\
\text { sum M }\end{array}$ & $p$ value \\
\hline Physical data & Age & 286 & 179 & 0.119022 \\
& BMI & 268.5 & 196.5 & 0.405742 \\
& Physical activity & 228.5 & 236.5 & 0.429622 \\
& (HPW) & & & \\
\hline Psychological & BDI & 211 & 254 & 0.129186 \\
tests & PSS & 212 & 253 & 0.140011 \\
\hline Short-term HRV & Respiratory rate & 260 & 205 & 0.632606 \\
& HR mean & 277 & 188 & 0.236113 \\
& SD1* & 191 & 274 & 0.018838 \\
& SDNN** & 176 & 289 & 0.002956 \\
& TP** & 178 & 287 & 0.003863 \\
\hline DBT & E-I* & 191 & 274 & 0.018838 \\
& HR mean & 281 & 184 & 0.176683 \\
& SD1** & 180 & 285 & 0.005016 \\
& SDNN** & 177 & 288 & 0.003382 \\
\hline
\end{tabular}

${ }^{*} p<0.05 ; * * p<0.01$.

DBT, deep breathing test; HR, heart rate; TP, total power; BMI, body mass index; HRV, heart rate variability; SDNN, SD of all normal RR intervals; BDI, Beck's Depression Inventory; PSS, Perceived Stress Scale. migraine patients was reported to be increased, while HF was found to be reduced, reflecting reduced parasympathetic activity [17].

Apart from disease-specific abnormalities of cardiovagal and sympathetic functions, both disorders - epilepsies and migraine - are associated with a wide range of psychiatric comorbidities, and stress is a prevalent migraine trigger [18]. Patients with unusually high respiratory rates at rest and subsequent lower parasympathetic activity might negatively influence the pathophysiology of their underlying disease [19]. Shifting the autonomic balance toward a more parasympathetic state by relaxation techniques and breathing exercises might be a successful therapeutic approach in such patients [20].

As results from repeated measures have shown that HRV can change due to short-term habituation [21], we compared HRV parameters during ascending periods of paced breathing with HRV during descending breathing periods in our patient groups. Apart from a minimal reduction of SDNN in the descending compared to the ascending breathing period, HR and HRV parameters yielded a lack of habituation over time in both patient groups. The minimal reduction of only SDNN but no further HRV parameters in the descending compared to the ascending breathing period obviously reflects a stabilizing effect of paced breathing on the patients' vigilance and attention during the repetition.

The small number of patients in our study limits the interpretation of HRV results. Disease severity, anticonvulsive medication, particularly carbamazepine, and migraine prophylactic treatment, especially with metopro- 
lol, might also influence autonomic cardiovascular modulation. Four of the 16 patients (25\%) with epilepsies received either carbamazepine or lacosamide at the time of HRV measurements, and 2 of 14 patients (14.3\%) in the migraine group received metoprolol, a substance clearly associated with side effects on the cardiac conduction system.

Even though the majority of our patients did not receive medications that have been unequivocally linked to HRV alterations, the observed differences between epileptic and migraine patients must be interpreted with caution, and further studies are needed to differentiate between the impact of drug effects and disease-related impairment of HRV more accurately.

In summary, our study is the first to find broad variation in spontaneous respiratory rates in 2 groups of patients with neurological diseases common in outpatients, as previously reported by HRV studies on healthy subjects. The observed negative correlation between respiratory rates and HRV suggests that simultaneous measure- ment of breathing rates can provide important additional information for resting HRV analyses. The larger reduction of HRV parameters with slow compared to fast breathing in our group of epileptic patients points toward possible dysfunction within the cardiovagal circuitry. Further studies with larger patient numbers and healthy controls are needed to confirm possible differences in $\mathrm{HRV}$ at rest and during deep breathing between epileptic and migraine patients.

\section{Acknowledgments}

The authors thank all subjects for their time, effort, and willingness to participate in the study.

\section{Disclosure Statement}

The authors declare that they have no conflicts of interest to disclose.

\section{References}

1 Sevcencu C, Struijk JJ. Autonomic alterations and cardiac changes in epilepsy. Epilepsia. 2010 May;51(5):725-37.

2 Yoshida S, Tanaka H, Mizutani M, Nakao R, Okamoto N, Kajiura M, et al. Autonomic nervous system function in adolescent migraineurs. Pediatr Int (Roma). 2017 Sep; 59(9):991-5.

3 Low PA, Tomalia VA, Park KJ. Autonomic function tests: some clinical applications. J Clin Neurol. 2013 Jan;9(1):1-8.

4 Assessment: Clinical autonomic testing report of the Therapeutics and Technology Assessment Subcommittee of the American Academy of Neurology. Neurology. 1996 Mar;46(3):873-80.

5 Vaschillo EG, Vaschillo B, Lehrer PM. Characteristics of resonance in heart rate variability stimulated by biofeedback. Appl Psychophysiol Biofeedback. 2006 Jun;31(2):129-42.

6 Song HS, Lehrer PM. The effects of specific respiratory rates on heart rate and heart rate variability. Appl Psychophysiol Biofeedback. 2003 Mar;28(1):13-23.

7 Ciccone AB, Siedlik JA, Wecht JM, Deckert JA, Nguyen ND, Weir JP. Reminder: RMSSD and SD1 are identical heart rate variability metrics. Muscle Nerve. 2017 Oct;56(4):674-8.

8 Bigger JT Jr, Kleiger RE, Fleiss JL, Rolnitzky LM, Steinman RC, Miller JP. Components of heart rate variability measured during healing of acute myocardial infarction. Am J Cardiol. 1988 Feb;61(4):208-15.
9 Bianchi A, Bontempi B, Cerutti S, Gianoglio P, Comi G, Natali Sora MG. Spectral analysis of heart rate variability signal and respiration in diabetic subjects. Med Biol Eng Comput. 1990 May;28(3):205-11.

10 Quintana DS, Elstad M, Kaufmann T, Brandt CL, Haatveit B, Haram M, et al. Resting-state high-frequency heart rate variability is related to respiratory frequency in individuals with severe mental illness but not healthy controls. Sci Rep. 2016 Nov;6(1):37212.

11 Electrophysiology TF; Task Force of the European Society of Cardiology and the North American Society of Pacing and Electrophysiology. Heart rate variability: standards of measurement, physiological interpretation and clinical use. Circulation. 1996 Mar;93(5): 1043-65.

12 Widjaja D, Caicedo A, Vlemincx E, Van Diest I, Van Huffel S. Separation of respiratory influences from the tachogram: a methodological evaluation. PLoS One. 2014 Jul: 9(7):e101713

13 Gilfriche P, Arsac LM, Daviaux Y, Diaz-Pineda J, Miard B, Morellec O, et al. Highly sensitive index of cardiac autonomic control based on time-varying respiration derived from ECG. Am J Physiol Regul Integr Comp Physiol. 2018 Sep;315(3):R469-78.

14 Lotufo PA, Valiengo L, Benseñor IM, Brunoni AR. A systematic review and meta-analysis of heart rate variability in epilepsy and antiepileptic drugs. Epilepsia. 2012 Feb;53(2): $272-82$.
15 Miglis MG. Migraine and Autonomic Dysfunction: Which Is the Horse and Which Is the Jockey? Curr Pain Headache Rep. 2018 Feb;22(3):19.

16 Gass JJ, Glaros AG. Autonomic dysregulation in headache patients. Appl Psychophysiol Biofeedback. 2013 Dec;38(4):257-63.

17 Matei D, Constantinescu V, Corciova C, Ignat B, Matei R, Popescu CD. Autonomic impairment in patients with migraine. Eur Rev Med Pharmacol Sci. 2015 Oct;19(20):3922-7.

18 Minen MT, Begasse De Dhaem O, Kroon Van Diest A, Powers S, Schwedt TJ, Lipton R, et al. Migraine and its psychiatric comorbidities. J Neurol Neurosurg Psychiatry. 2016 Jul;87(7): 741-9.

19 Gass JJ, Glaros AG. Autonomic dysregulation in headache patients. Appl Psychophysiol Biofeedback. 2013 Dec;38(4):257-63.

20 de Bruin EI, van der Zwan JE, Bögels SM. A RCT Comparing Daily Mindfulness Meditations, Biofeedback Exercises, and Daily Physical Exercise on Attention Control, Executive Functioning, Mindful Awareness, Self-Compassion, and Worrying in Stressed Young Adults. Mindfulness (N Y). 2016;7(5):118292.

21 Feda DM, Roemmich JN. Effect of interpersonal and cognitive stressors on habituation and the utility of heart rate variability to measure habituation. Stress Health. 2016 Oct; $32(4): 320-7$. 